\title{
PREVALÊNCIA DE OBESIDADE NA INFÂNCIA EM DIFERENTES AGRUPAMENTOS SOCIAIS E AIMPORTÂNCIA DE ESTRATÉGIAS PEDAGÓGICAS
}

\section{Prevalence of obesity in childhood in different social groups and the importance of teaching strategies}

\author{
Adriana Berleze ${ }^{l}$; Leris Salete Bonfanti Haeffner ${ }^{2}$, Nadia Cristina Valentini ${ }^{3}$
}

\begin{abstract}
RESUMO
Esta pesquisa investigou a prevalência de obesidade de crianças, entre 6 a 8 anos de idade, da $1^{\text {a }}$ série do Ensino Fundamental, em diferentes agrupamentos sociais, da cidade de Santa Maria-RS. Participaram deste estudo 424 crianças (212 meninas e 212 meninos) distribuídas nos agrupamentos 1 (região central, classe média), 2 (região periférica, classe média), e 3 (região periférica, classe pobre). Peso e estatura foi medida em cada sujeito. Sobrepeso e obesidade foi definida pelo índice de massa corporal (IMC, $\mathrm{kg} / \mathrm{m} 2$ ) do percentil 85 a 95 e > 95, respectivamente. A referência utilizada foi do NCHS. Para a análise dos dados utilizou-se a estatística descritiva e chi2 $(\mathrm{p}<0,05)$. Os resultados mostram uma prevalência de obesidade de $29,95 \%$ na amostra e semelhança entre agrupamentos e gêneros. A presença de sobrepeso e obesidade no início da idade escolar reforça a importância de hábitos saudáveis na infância, tanto na alimentação como na prática sistemática de atividades motoras.
\end{abstract}

Palavras- Chave: obesidade, IMC, criança, estratégias pedagógicas.

\section{SUMMARY}

The subject of this research was the prevalence of obesity in children, from 6 to 8 years old attending the first-grade of 'Fundamental Teaching', in different social groups, in Santa Maria, RS. The study included 424 children (212 girls and 212 boys) distributed in the following groups: group 1 (central area, middle class), group 2 (suburban area, middle class), and group 3 (suburban area, poor class). Height and weight were measured in all the children. Overweight and obesity was defined by body mass index (BMI, $\mathrm{kg} / \mathrm{m} 2$ ) of $85-95$ th percentile and $>95$ th percentile, respectively, according to the NCHS. Data are reported as frequencies and compared by chi2 test $(\mathrm{p}<0,05)$. The prevalence of obesity in the population examined was $29,95 \%$, with no differences among the social groups and gender. The presence of overweight and obesity in children first starting at school reinforces the importance of healthy habits in the childhood, including healthy eating and the systematic practice of physical activities.

Keywords: obesity, BMI, child, teaching strategic.

\section{INTRODUÇÃO}

A trajetória do desenvolvimento da criança é caracterizada por mudanças de comportamento no decorrer da vida. Através de uma visão dinâmica, essas mudanças ocorrem direcionadas por restrições individuais; por experiências vivenciadas pelo indivíduo - restrições do contexto; e pela especificidade e complexidade das atividades vivenciadas - restrições da tarefa. Nessa perspectiva, a preocupação em saber qual a natureza, inata ou adquirida, que determina a maneira como a criança cresce e se desenvolve, passa a ser até mesmo pouco relevante. A maioria dos teóricos contemporâneos busca investigar as interações entre os diversos fatores que influenciam o desenvolvimento ${ }^{1,2,3,4}$; pois, se a hereditariedade determina o potencial humano, é o meio que determina até onde o ser humano pode chegar ${ }^{5}$.

Essas interações (hereditárias e ambientais) acabam influenciando nas principais mudanças da criança, ocasionando o desenvolvimento da mesma em ritmos e intensidades diferentes. No entanto, essas transformações parecem estar atreladas, principalmente, ao desenvolvimento físico, social e motor na infância. No aspecto físico, é nas transformações de crescimento como o peso e a estatura, que se pode tornar

Trabalho realizado na Universidade Federal de Santa Maria - UFSM

${ }^{1}$ Adriana Berleze- Doutor Ciëncias do Movimento Humano- Universidade Federal do Rio Grande do Sul - UFRGS

${ }^{2}$ Leris Salete Bonfanti Haeffner- Doutor em Medicina - Universidade Federal de Santa Maria - UFSM

${ }^{3}$ Nadia Cristina Valentini- PhD. Comportamento Motor- Professor da Universidade Federal do Rio Grande do Sul - UFRGS 
possível a organização do conceito de imagem corporal, onde sentimentos sobre o próprio corpo podem afetar as preferências nas atividades. No aspecto social, a infância se caracteriza por um período de acentuadas mudanças de comportamento e atitudes, as quais sofrem forte influência pelas respostas dos outros, principalmente das pessoas que são significativas para esta criança em desenvolvimento. No aspecto motor, novas experiências surgem na infância, como por exemplo, prontidão para dominar novas habilidades (caminhar, correr, saltar, chutar, arremessar, quicar, entre outras). O aprimoramento dessas habilidades é conseqüência da variedade de experiências que pode ser oportunizada a criança, pois se acredita que estímulos dados, no início da infância, novos comportamentos motores, cognitivos e sociais serão alcançados ${ }^{6,7}$.

Salienta-se, que no decorrer do desenvolvimento as crianças podem evidenciar atrasos. Esses atrasos dependem de muitos fatores, sejam eles de ordem genética, endócrina e/ou ambiental. Tanto os fatores biológicos como ambientais podem modificar o curso das mudanças e, embora o enfoque de pesquisas se volte para fatores biológicos ${ }^{8}$, os fatores ambientais têm se mostrado determinantes para o desenvolvimento na criança e um estilo de vida saudável da mesma ${ }^{5,7}$. Um contexto cercado por uma escassez de movimentação infantil, onde o envolvimento dos pais com seus filhos é cada vez menor, uma alimentação rica em gordura, pobre em fibras, os crescentes hábitos de assistir à televisão e ficar ao computador, podem transformar as crianças em pequenos obesos, caracterizando a obesidade infantil como uma verdadeira epidemia ${ }^{9}$.

Uma transição nutricional, caracterizada pela redução de déficits nutricionais e o aumento de prevalência de obesidade, tem sido observada no Brasil, nas mais variadas faixas etárias. Os dados da obesidade no Brasil têm se mostrado preocupantes, principalmente na infância e adolescência, tanto que este tema tornou-se o foco de várias pesquisas brasileiras recentemente publicadas $^{10,11,12,13}$. Silva, Balaban e Motta ${ }^{10}$ comparando a prevalência de sobrepeso e obesidade em um grupo de préescolares, escolares e adolescentes de diferentes regiões socioeconômicas, constataram que em uma amostra de 1616 crianças e adolescentes, foram encontrados $14,5 \%$ dos escolares com sobrepeso e 8,3\% dos escolares com obesidade. Garlipp et $\mathrm{al}^{11}$ evidenciaram tendência semelhante quanto aos níveis de obesidade. Em um estudo que procuraram descrever o estado nutricional de crianças e adolescentes de 7 a 14 anos de idade do estado do Rio Grande do Sul, constataram que 18,9\% de meninos e $18,3 \%$ de meninas estão com sobrepeso. Giugliani e Carneiro ${ }^{12}$ ao avaliar o estado nutricional de 452 escolares, observaram a prevalência de sobrepeso e obesidade de $21,1 \%$ nos meninos e $22,9 \%$ nas meninas. Nessa mesma direção, Poletto ${ }^{13}$ investigando um total de 777 escolares, entre 7 a 14 anos de idade, da Rede Municipal de Ensino de Porto Alegre constatou que 22\% dos escolares se encontram com prevalência de obesidade.

Esse aumento de obesidade pode estar relacionado com vários fatores, como por exemplo, um contexto cercado por uma escassez de movimentação, uma alimentação rica em gordura, a violência nas grandes cidades (fazendo com que as pessoas façam à opção de usar transporte motorizado para se locomover) e os crescentes hábitos de assistir à televisão e ficar ao computador ${ }^{12,9,14}$. Embora os múltiplos fatores da obesidade infantil possam resultar de causas internas (fatores genéticos ou metabólicos), causas externas (problemas psicológicos, alimentares, e de inatividade), como também, refletirem a complexa interação entre os fatores internos e externos (como os aspectos socioeconômicos, culturais e comportamentais), sem dúvida alguma a obesidade tem maior relação com a baixa freqüência de atividade física ${ }^{9}$ A Organização Mundial da Saúde relaciona a obesidade infantil com níveis muito baixos de atividade e aptidão física ${ }^{15} \mathrm{e}$ estudos clínicos ${ }^{16}$ reforçam essa relação, demonstrando que $67,5 \%$ dos casos de obesidade estão associados à inatividade.

Dessa forma, devido à preocupação com os índices crescentes de obesidade infantil, o presente estudo objetivou investigou a prevalência de obesidade de crianças, entre 6 a 8 anos de idade, da $1^{a}$ série do Ensino Fundamental, em diferentes agrupamentos sociais, da cidade de Santa Maria-RS. Para o presente estudo as seguintes hipóteses foram estabelecidas: (1) diferenças de prevalência de obesidade são esperadas para os agrupamentos sociais; (2) igualdade na prevalência de obesidade é esperada entre os gêneros.

\section{MATERIAIS E MÉTODOS}

\section{Caracterização e universo da pesquisa}

Esta pesquisa caracterizou-se como sendo descritiva, não se restringindo unicamente em expor e explicar o acontecido, mas ao considerar a realidade sugerir estratégias que contribuam para a atual prática ${ }^{17,18}$. Fizeram parte da mesma, crianças de 6 a 8 anos de idade, da $1^{\text {a }}$ série do Ensino Fundamental, da cidade de Santa Maria - RS. Para o agrupamento dos escolares seguiram-se princípios propostos por Hartshorne ${ }^{19}$, ou seja, os limites de uma inter-relação e a verificação e delimitação de um fato característico de uma porção territorial, bem como a consideração dos objetivos específicos de cada pesquisador. No presente estudo o agrupamento dos bairros foi realizado por meio da proximidade geográfica e de características socioeconômicas similares entre eles, configurando-se da seguinte forma: a) agrupamento 1região central- classe média: bairros que apresentam uma infraestrutura urbana satisfatória, não apresentando problemas de saneamento básico e infra-estrutura, possuem grandes construções, vias bem pavimentadas com asfalto, fornecimento adequado de energia elétrica e água, o fluxo de veículos é intenso, a maior parte da população tem o melhor poder aquisitivo do 
município; b) agrupamento 2- região periférica- classe média: são bairros, que nos últimos anos, vem apresentando considerável desenvolvimento, com uma infra-estrutura regular, ruas com bom calçamento de pedras irregulares e saneamento básico; c) agrupamento 3- região periférica- classe pobre: a maioria dos moradores possui baixo poder aquisitivo, há predomínio de operários e subempregados, com parcela considerável de desempregados. Na sua infra-estrutura, as ruas do bairro, são na maioria, calçadas com pedras irregulares, porém muitas ruas são desprovidas de calçamento e/ou asfaltamento ${ }^{20,21}$.

\section{Seleção da amostra}

O tamanho mínimo da amostra representativo de cada agrupamento foi definido utilizando-se a fórmula de população finita com margens de erro de 5\%. A amostra foi constituída de 424 crianças (212 meninas e 212 meninos), distribuídas proporcionalmente nos agrupamentos e em cada escola. As escolas foram sorteadas por amostragem aleatória simples, de maneira casual, mas com o critério de que pelo menos uma escola de cada Rede de Ensino fizesse parte de cada agrupamento. O sorteio das crianças nas escolas foi realizado de forma estratificada proporcional; sendo que, para cada turma, foi selecionada uma amostra aleatória proporcional entre os gêneros. O Termo de Consentimento Informado foi obtido de todos os participantes do presente estudo.

\section{Instrumentos}

Os dados coletados para avaliar o estado nutricional foram idade, peso e estatura, tendo-se utilizado balança eletrônica portátil digital, estadiômetro de madeira e fichas de registro. O diagnóstico da obesidade foi feito por meio do IMC (Índice de Massa Corporal), tendo como referência o NCHS (National Center for Health and Statistics) ${ }^{22}$.

\section{Procedimentos para coleta dos dados}

Os dados foram coletados nas escolas com a autorização prévia das Secretarias Municipais e Estaduais, e das escolas particulares, durante três meses. A coleta foi realizada por regiões, começando pela região leste da cidade, estendendo-se para as regiões sul, central e norte, e finalizando com a região oeste.

Para a avaliação antropométrica as crianças apresentaramse descalças e vestidas com roupas leves. Para a coleta do peso, as crianças permaneceram em pé sobre a plataforma da balança, com os braços ao longo do corpo, em uma atitude tranquiila. $\mathrm{O}$ peso foi registrado em quilogramas $(\mathrm{kg})$ e gramas $(\mathrm{g})$. O valor encontrado foi registrado em uma ficha individual para cada criança. Para a coleta da estatura, as crianças posicionaram-se de pé sobre uma superfície de madeira, de costas para a escala métrica, com os pés paralelos e os tornozelos unidos. Assegurou-se que as nádegas, os ombros e a parte posterior da cabeça estivessem tocando a régua, e os braços permanecessem soltos ao longo do corpo. Com a mão sob o queixo da criança, posicionou-se sua cabeça de forma que a parte inferior da órbita ocular permanecesse no mesmo plano do orifício externo do ouvido. Baixou-se lentamente a extremidade móvel do aparelho (cursor) até tocar o topo da cabeça em sua parte média, sem empurrar a cabeça para baixo. Fixou-se o cursor, e feita a leitura da régua até o milímetro mais próximo, registrouse o escore.

\section{Análise dos dados}

A análise dos resultados quanto à obesidade foi realizada mediante a seguinte classificação: crianças com sobrepeso aquelas que tinham o IMC entre o percentil 85-95 e obesas as com o percentil $\geq 95^{22}$. Os pontos de corte utilizados de acordo com a idade dos escolares, segundo referência do $\mathrm{NCHS}^{22}$, foram, respectivamente, 6 anos: $17,01 \mathrm{Kg} / \mathrm{m}^{2}$ (meninas) e $16,91 \mathrm{Kg} / \mathrm{m}^{2}$ (meninos); 7 anos: $17,40 \mathrm{Kg} / \mathrm{m}^{2}$ (meninas) e $17,09 \mathrm{Kg} / \mathrm{m}^{2}$ (meninos); e 8 anos: $17,95 \mathrm{Kg} / \mathrm{m}^{2}$ (meninas) e $18,31 \mathrm{Kg} / \mathrm{m}^{2}$ (meninos).

Para análise dos resultados foi usada estatística descritiva, calculando-se a distribuição de freqüência e $\mathrm{Chi}^{2}$ com nível de significância de 0,05. Para a análise de estatística foram utilizados os programas Epi-Info ${ }^{23}$ (versão 6,0) e Stata ${ }^{24}$ (versão 10,0).

\section{RESULTADOS}

\section{Prevalência de obesidade por agrupamento social}

Dentre as 424 crianças investigadas, o número total de obesos encontrado foi de 127 crianças, perfazendo um total de $29,95 \%$ da população investigada. A distribuição das crianças obesas foi $35,35 \%, 34,64 \%$ e $24,24 \%$, respectivamente por agrupamento $1,2 \mathrm{e} 3$, mas sem diferença estatística significante $(p=0,055)$. Esse resultado refuta a hipótese (1) do presente estudo, na qual se esperava uma prevalência diferenciada de obesidade entre os agrupamentos sociais. 
Tabela I-Distribuição de crianças obesas por agrupamento social

\begin{tabular}{ccccc}
\hline Agrupamentos & $\mathrm{N}$ & $\mathrm{n}$ & $\%$ & $\mathrm{p}$ \\
\hline $\begin{array}{c}\text { 1 Região central } \\
\text { classe média }\end{array}$ & 99 & 35 & 35,35 & \\
$\begin{array}{c}\text { 2 Região periférica } \\
\text { classe média }\end{array}$ & 127 & 44 & 34,64 & \\
$\begin{array}{c}\text { 3 Região periférica } \\
\text { classe pobre }\end{array}$ & 198 & 48 & 24,24 & \\
\hline TOTAL & 424 & 127 & & \\
\hline
\end{tabular}

$\mathrm{N}$ : número total de crianças

n: número de obesos

$\%$ : percentual de obesos por agrupamento

\section{Prevalência de obesidade por gênero}

Com relação ao gênero, observa-se (Tabela 2) que, das 127 crianças obesas encontradas, 62 são meninas (48,82\%); e 65 meninos $(51,18 \%)$. O percentual de obesidade encontrado foi de $14,62 \%$ para as meninas e $15,33 \%$ para os meninos, não existindo diferença significativa entre os grupos $(\mathrm{p}=0,089)$. Esses resultados suportam a hipótese (2) do estudo, no qual se esperava uma semelhança na prevalência de obesidade entre os gêneros.

Tabela 2- Distribuição de crianças obesas por gênero

\begin{tabular}{lllll}
\hline Gênero & N & $\mathbf{n}$ & \% & $\mathbf{p}$ \\
\hline & & & & 0,089 \\
Feminino & 212 & 62 & 14,62 & \\
Masculino & 212 & 65 & 15,33 & \\
\hline TOTAL & $\mathbf{4 2 4}$ & $\mathbf{1 2 7}$ & & \\
\hline
\end{tabular}

$\mathrm{N}$ : número total de crianças

n: número de obesos

$\%$ : percentual de obesos por agrupamento

\section{DISCUSSÃO}

Os resultados encontrados mostram percentuais elevados de crianças obesas, fato esse já reportado em pesquisas prévias ${ }^{10,11}$. Com relação à prevalência de obesidade por agrupamentos sociais este estudo mostrou resultados semelhantes a outros prévios ${ }^{10,13}$, os quais constaram que o sobrepeso e obesidade foram mais freqüentemente observados entre crianças e adolescentes de melhor condição socioeconômica. No entanto, a não observação de diferença estatística significante entre os grupos, combinadas com o elevado índice de $24,24 \%$ de obesidade no agrupamento 3 sugere que a ingesta de gordura e carboidratos, alimentos mais baratos, quando comparados com alimentos de origem protéica, pode ser o fator de influência nesses índices de obesidade de classes econômicas menos favorecidas ${ }^{14}$.

Quanto à obesidade entre os gêneros os resultados corroboram com pesquisas prévias ${ }^{11,12}$, no qual a prevalência de elevados índices de obesidade se manteve tanto para os meninos como para meninas. Cabe salientar, que um dos aspectos comportamentais evidentes, entre os 5 a 7 anos de idade, são as mudanças nutricionais. É na infância um dos grandes períodos para o desenvolvimento e manutenção da obesidade, pois aproximadamente $60 \%$ dos obesos aos 7 anos permanecem obesos quando adultos ${ }^{25}$.

Portanto, é neste período que as crianças devem estar ativamente envolvidas na descoberta diversificada de movimentos, bem como, na combinação desses movimentos, aplicando-os em diferentes tarefas e contextos educacionais. Os prováveis problemas constatados no início da escolaridade como a obesidade infantil e atrasos no desempenho motor, podem ser sanados com naturalidade com propostas pedagógicas apropriadas que acabam influenciando diretamente no engajamento da criança em vivências motoras nos períodos subseqüentes do seu desenvolvimento e, conseqüentemente criando hábitos saudáveis para toda a vida ${ }^{26,}$ 27,3

\section{CONSIDERAÇÕES FINAIS}

Finalizando a pesquisa, as considerações finais nos fazem refletir sobre o alto índice de obesidade encontrado. Tudo nos leva a acreditar que pode afetar qualquer nível social, pois esse índice apareceu nos três agrupamentos de forma expressiva. Esse crescimento parece seguir uma tendência de obesidade mundial, que, provavelmente, tenha como um dos fatores relacionados ao estilo de vida, como maus hábitos alimentares e pouco engajamento em atividade física. Naturalmente, esses hábitos já deveriam ter sido criados antes da criança entrar na idade escolar. Conduto, no momento em que a criança tem a escola como um novo microssistema de convívio, esta deve juntamente com o microssistema familiar, favorecer condições, de estrutura física e humana, incentivos e melhores oportunidades de atividades motoras infantis. Quando se trabalha com crianças obesas essas condições são muito mais importantes.

Nesta perspectiva, enfatiza-se que os professores de 
educação física são os responsáveis principais no desenvolvimento de programas motores de qualidade, adaptando uma variedade de características individuais como nível de desenvolvimento, experiências prévias de movimento e interesses. Porém, cabe salientar que não é o suficiente colocar a criança mais ativa, aumentando a quantidade e intensidade do programa de atividade física. Entretanto, propiciar as crianças aprendizagem dos conteúdos; o desenvolvimento de competências nas mais variadas formas de movimento; e experiências de valorização e pratica de atividades motoras de forma regular, incorporando hábitos saudáveis no decorrer do seu desenvolvimento, são estratégias pedagógicas que estimulam as crianças a dar continuidade ao engajamento nas atividades de forma prazerosa e consistente, educando-as assim para ter um estilo de vida ativo.

Para finalizar, cabe salientar que criança precisa de uma segura emocional e essa deve partir, principalmente, da família. Qualquer tentativa de melhorar a qualidade do desenvolvimento infantil, em termos melhorias nos parâmetros nutricionais, motores e psicossociais, teria que partir das mudanças dos hábitos familiares. Portanto, é necessário que o professor de Educação Física invista no potencial instigativo de suas aulas, estimulando as crianças a buscar parcerias com a família para construir uma prática motora sistemática. Desta forma, para que a aula de Educação Física alcance um dos seus maiores objetivos, que é estimular o engajamento motor da criança, cabe ao profissional da área interagir cada vez mais com a família.

\section{REFERENNCIAS BIBLIOGRÁFICAS}

1. Newell K. Constraints on the development of the coordination. In: Wade M.; Whiting, HTA. (Eds). Motor Development in children: aspects of control and coordination. Dordrecht: Martinus Nijhof, 1986.

2. Bronfenbrenner U. A ecologia do desenvolvimento humano: experimentos naturais e planejados. $1^{\mathrm{a}} \mathrm{ed}$. Porto Alegre: Artes Médicas, 1996.

3. Gallahue D. Ozmun JC. Compreendendo o Desenvolvimento Motor: Bebês, Crianças, Adolescentes e Adultos. São Paulo: Phorte Editora, 2001.

4. Haywood KM; Getchell N. Desenvolvimento motor ao longo da vida. $3^{\mathrm{a}}$ ed. Porto Alegre: Artmed Editora, 2004

5. Thomas MR. Comparing theories of child development. $4^{\mathrm{a}}$ ed. Pacific Grove: Books Cole. 1995.

6. Bee H. A Criança em desenvolvimento. 7a. ed. Porto Alegre: Artes Médicas, 1996. cap. 16. p. 452-475.

7. Papalia DE; Olds SW. Desenvolvimento Humano. 7a ed. Porto Alegre: Artes Médicas Sul, 2000.

8. Clark JE; Whitall J. What is Motor Development? The Lessons of History. Quest, 41: 183-202, 1989.
9. Damaso A. Nutrição e exercício na prevenção de doenças. $1^{\text {a }}$ ed. Rio de Janeiro: Medsi, 2001

10. Silva GAP; Balaban G; Motta MEF. Prevalência de sobrepeso e obesidade em crianças e adolescentes de diferentes condições socioeconômicas. Revista Brasileira Saúde Materno Infantil, 2005. 5(1):53-59.

11. Garlipp D; Bergmann G; Lorenzi T; Marques AC; Gaya A; Torres L. et al. Avaliação do estado nutricional de crianças e adolescentes do estado do Rio Grande do Sul através do índice de massa corporal. Revista Perfil, 2005. 7(7): 48-52

12. Giugliani R; Carneiro EC. Fatores associados à obesidade em escolares. Jornal de Pediatria, 2004. 80, 1: 17-22.

13. Poletto AR. Hábitos de Vida, Estado Nutricional, Perfil de Crescimento e Aptidão Física Referenciada à Saúde: Subsídios para o Planejamento de Educação Física e Esportes na Escola Cidadã. Dissertação de Mestrado. Universidade Federal do Rio Grande do Sul. 2001. 160p.

14. Guedes DP; Guedes J. ERP. Controle de peso corporal: composição corporal, atividade física e nutrição. $1^{\mathrm{a}}$ ed. LondrinaParaná: Midiograf,1998. cap. 1. p. 11-43.

15. Colavitti F. Epidemia de gordura. Revista Galileu [periódico on line]. 2004. Disponível em <http://www revistagalileu.globo.com [2004 set. 12] 160 (10).

16. Lancha AHJr. Obesidade: uma abordagem multidisciplinar. Ed. Guanabara Koogan, 2006.

17. Gill CA. Métodos e técnicas de pesquisa social. $5^{\text {a }}$ ed. São Paulo: Atlas, 1999.

18. Thomas J; Nelson J. Métodos de pesquisa em atividade física. $3^{\mathrm{a}}$ ed. Ed. Artmed, 2002.

19. Hartshorne R. Propósitos e natureza da geografia. $2^{\mathrm{a}}$ ed. São Paulo: Hucitec/EDUSP, 1978.

20. Corrêa RL. Região e organização espacial. $2^{a}$ ed. São Paulo: Ática, 1987.

21. Farinha TM. Mapeamento das causas de óbitos por bairros no distrito sede de Santa Maria-RS. [Monografia de Especialização- Programa de Pós-Graduação em Interpretação de Imagens Orbitais e Suborbitais]. Santa Maria (RS): Universidade Federal de Santa Maria; 1999.

22. NCHS - National Center for Health and Statistics. Growth curves for children birth-18 years. Vital and health statistics. DNEW publ, (PHS). Série, 165, 1978.

23. Dean AG; Dean, JA; Coulombier D; et al. Epi Info- Um sitema de processamento de texto, banco de dados e estatística para Epidemiologia em Microcomputadores. Trad. de Marilda Lauretti da Silva Guedes. Atlanta, centers of Diease Control and Prevention (CDC), 1994.

24. Stata Corporation. Stata Statistical Software: release 10,0. College Station, 2006.

25. Costa, Maria CO; Souza Ronald P. de (ORG.). Adolescência aspectos clínicos e psicossociais. Porto Alegre: Artmed, 2002. 
$464 \mathrm{p}$.

26. Clark J. Motor development. In: Ramachandran VS. (Ed.), Encyclopedia of human behavior. (vol. 3, pp.245-255). New York: Academic Press, 1984.

Endereço para correspondência:

Adriana Berleze

Rua João da Fontoura e Souza, 100/ apto. 203. Bairro Camobi

Santa Maria -RS CEP: 97105-210

Email: adrianaberleze@yahoo.com.br

Fone: (55) 9114-0233
27. Gallahue D. Understanding Motor Development: infants, children, adolescents. Benchmark Press. Indianapolis, Indiana 2 nd edition, 1989. 